\title{
Effects of Essential Oils and Aromatic Plants on Alzheimer's Disease and Dementia
}

\author{
Nicha Hemangkorn, Pamonphon Phummai, Patranan Punyacharoen
}

Mahidol University International Demonstration School, Thailand

Corresponding Author: Nicha Hemangkorn

\begin{abstract}
The primary objective of this research was to investigate how beneficial essential oils and aromatic plants are at curing Alzheimer's disease and dementia. Alzheimer's Disease (AD) is known to be one of the most incurable prevalent diseases in the elderly. Agitation, cognitive impairments, communication difficulty, irritability, and confusion may advance in $\mathrm{AD}$ patients. Even though the behavioral causes contributing to the disease have not yet been discovered, aggregation of tau and amyloid proteins is found in the brain of patients with Alzheimer's. With numerous experiments conducted on these proteins, scientists have surmised that this accumulation is a causative factor of Alzheimer's disease. Multiple scientists and experts have carried out several studies regarding the enlargement of amyloid plaques on Alzheimer's. In addition, the effects of aromatherapy, including divergent results of alternative essential oils, have been analyzed. This paper examines the distinctions between Alzheimer's disease and dementia. The review elaborates on the causes and the symptoms before describing the effects of essential oils and aromatic plants. Subsequently, this provides rudiments regarding the benefits and hazards of applying essential oils and aromatic plants to treat either Alzheimer's disease or dementia. This study focuses on utilizing essential oils and aromatic plants in the medical profession as an alternative treatment for Alzheimer's disease and dementia. Likewise, a couple of case studies are shown in the paper with the analysis of amyloid plaques in mice and the effectiveness of lavender, rosemary, and orange essential oils on participants.
\end{abstract}

Keywords: Alzheimer's disease Dementia Essential oils Aromatic plants Aromatherapy Amyloid and Tau

\section{INTRODUCTION}

\subsection{Characteristics of Alzheimer's disease}

Alzheimer's disease (AD) is a brain disease that causes memory, cognitive, and behavioral issues. Symptoms of Alzheimer's disease generally develop in individuals in their mid-60s (NIH National Institute on Aging [NIA], 2021). Brain cells may be deteriorating if the brain undergoes memory loss, disorientation, or other significant changes in the ways our mind operates. The most prevalent early indications of Alzheimer's are difficulty in memorizing and building up new skills. Alzheimer's disease causes progressively severe symptoms as it proceeds through the brain, including unsubstantiated fear about family, friends, and personal caretakers and hallucinations (Alzheimer's Association, n.d.; NIH National Institute on Aging [NIA], 2017).

The primary cause of Alzheimer's disease has not been scientifically proven. However, the researchers have found an abnormal accumulation of proteins, triggering the loss of function of neurons in the brain over a division of time (NHS, 2021; NIH National Institute on Aging [NIA], 2017).

Alzheimer's is the most widespread cause of dementia, referring to memory loss and other cognitive impairments that intervene with everyday life (NIA, 2021). Besides, other conditions may be developed 
through the ability to communicate and comprehend (Leonard, 2018).

\subsection{The effectiveness of aromatherapy on Alzheimer's disease}

Aromatherapy utilizes the essential oils obtained from plants as a supplementary and alternative medicine. However, typical aroma massage, aroma baths, and other forms are not mentioned in the medical treatment shown in the modern study of aromatherapy (Jimbo, Kimura, Taniguchi, Inoue, Urakami, 2010). Nonetheless, the healing process should not be substituted by the essential oils, only to ensure patients' calmness and satisfaction (Dementia specialist Admiral Nurses, 2019).

The olfactory receptors are activated by aromatherapy which triggers the region of the brain associated with emotions, comforting and alleviating anxiety and depression symptoms. Numerous studies illustrate that aromatherapy can assist individuals with dementia, including Alzheimer's disease (AD) (Laguipo, 2021). Oils in the treatment, utilizing with Alzheimer's disease (AD), consist of lavender and orange oil which stimulates the parasympathetic nervous system, together with a rosemary and a lemon oil to soothe depression and anxiety (Jimbo, Kimura, Taniguchi, Inoue, \& Urakami, 2010).

Besides, aromatherapy is increasingly becoming a prominent supplementary treatment for dementia patients (Laguipo, 2021). Consequently, it can assist individuals with $\mathrm{AD}$ to improve their cognitive function by relieving agitation, restlessness, and insomnia (Jimbo, Kimura, Taniguchi, Inoue, Urakami, 2010).

\subsection{Essential oils and their therapeutic properties}

Scientific evidence has proved that essential oils, for instance, lavender, bergamot, and lemon balm, contribute to a dementia patient's aggression, agitation, and other psychosis (Laguipo, 2021). Besides, there are two ways of extracting essential oils from plants: distillation and expression.
In distillation, the compounds from plants are heated and released in the form of gas. As the vapor travels through the condenser, steam is subsequently condensed back into water. The oil then rises to the top after it cools down. On the other hand, heat is not required when extracting oil by expression. Instead, the mechanical pressure causes the oil to proceed and generate citrus oils (Upham, 2020).

During the inhalation, fragrance molecules in essential oil diffuse from the olfactory nerve to the amygdala, the brain's emotional center (Johns Hopkins Medicine, n.d.).

\subsection{Complementary treatments for Alzheimer's by aromatic plants}

Essential oils are extracted from various aromatic plant components such as flowers, barks, stems, leaves, roots, etc., which have been documented to be used in aromatherapy (Babar Ali-Firoz Anwar et al., 2015). Furthermore, sensory gardens can provide patients' pleasant smells, stimulating memory centers in the brain. By triggering this center, lost memories could be recalled (Five Star Senior Living, 2020).

Lavender (Lavandula), one of the typical aromatic plants, is well-known as the mother of all oils. Lavender has been proven in various studies regarding the improvement in dementia's behavior. Additionally, Agitation, one dementia and Alzheimers' characteristic, can be alleviated by lavender scent. Aside from lavender, lemon balm (Melissa officinalis) could lessen strain and anxiety in AD patients (Upham, 2020). It is also predicted to boost memory and thinking skills. However, the evidence has not been scientifically supported for some of the uses (Natural Medicines Comprehensive Database Consumer Version, n.d.). Furthermore, men's cologne and deodorant commonly contain bergamot (citrus bergamia), an essential oil that can aid individuals who are tense, apprehensive, or depressed (Upham, 2020). 
2. The distinctions between Alzheimer's and dementia, and fundamentals of Alzheimer's disease.

\subsection{Differences between ALzheimer's and dementia}

In elderly people, the most frequent cause of dementia is Alzheimer's disease. Dementia is characterized by a loss of cognitive abilities, for instance, memorizing, thinking, and logically analyzing. In addition, patients' everyday routines are interrupted by their abnormal physiological capability (NIA, 2021).

Dementia is not a disease but rather a syndrome. Multiple types of dementia, known as mixed dementia, can occur at once in which patients have several conditions. An autopsy is the only way to verify mixed dementia. (The Healthline Editorial Team, 2020).

In contrast, Alzheimer's disease alters brain functions that are in charge of conducting thoughts, memories, and languages. However, apart from affecting mental function, there is also a chance of damaging physical function. For instance, the bowel and bladder are physically affected, resulting in signs and symptoms of the disease. The symptoms of Alzheimer's often develop gradually. However, each patient's behavior changes and the time interval for the symptoms to build may vary. During the early stages of Alzheimer's disease, patients may experience anxiety, irritability, lack of creativity, mild obliviousness, complications regarding time and location, and conversation difficulty (Southern Cross Medical Library, 2020).

\subsection{Rudiments of Alzheimer's disease}

Chemical messengers, known as neurotransmitters, are responsible for transmitting messages or signals between brain cells, which declines when amyloid and tau damage brain cells. Patients with Alzheimer's, therefore, usually contain low levels of one neurotransmitter in the brain. Various areas in the brain are weakened, particularly the first area where memories are stored. Accordingly, alternative regions are affected in rare cases. Nevertheless, the earliest symptoms that appear frequently are problems associated with vision or language (NHS, 2021).

It is hypothesized that Amyloid$\beta(\mathrm{A} \beta)$ aggregates are presented in the brain at the initial stage of Alzheimer's disease; however, elderly patients are rarely diagnosed by brain biopsy (LeinonenAlafuzoff, 2010). Interruption of betaamyloid accumulation occurs among the communicating cells and triggers immunity leading to an inflammatory response; hence, brain cells deteriorate (Alzheimer's Association, 2017).

Tau is known as a protein found inside the neurofibrillary tangles, which are insoluble twisted fibers located in the brain's cells. It has a vital role in forming part of the microtubules. These tubules carry nutrients and other essential substances from one nerve cell to another. Despite its favorable function, the tau protein is aberrant in Alzheimer's disease, disintegrating microtubules to collapse (BrightFocus Foundation, 2019).

Alzheimer's disease causes the brain cells to degenerate, lose connection, and ultimately die. However, there is still an ongoing controversy about whether amyloid and tau cause disease or are just a part of disease progression (Southern Cross Medical Library, 2020).

The hippocampus and the entorhinal cortex are the first two brain regions being exposed to Alzheimer's disease (NIA, 2021). As a result, patients with Alzheimer's tend to have their memories deteriorated. One hundred billion nerve cells are associated with other cells, which operate communication networks inside the brain. Alzheimer's has been admitted by scientists that the disease prevents the braina tiny factory-from its regular function. Despite the genuinely existing causes being unknown, like an actual factory, damages from Alzheimer's induces issues in other parts of the human body. The brain is irreversibly affected, cells lose their 
functions, and subsequently die (Alzheimer's Association, n.d.).

To make a diagnosis, medical practitioners inquire individuals about their overall health; for instance, the use of prescription and over-the-counter medicines, dietary, or perhaps even their preceding medical issues, capacity to do everyday activities, and changes in demeanor and disposition. Arrange the tests concerning memory, problem-solving, attention, counting, and language. To point out all possible causes of the illness, run standard medical tests, for example, blood and urine tests. Utilize brain scans to verify an Alzheimer's diagnosis or eliminate other possible causes, for instance, computed tomography (CT), magnetic resonance imaging (MRI), or positron emission tomography (PET) (NIA, 2021).

\section{Benefits and Hazards of Essential oils and Aromatic plants}

In the present day, therapeutic plants and their elements have contributed as a supplement to alternative medicine for medical treatment. Plants, aromatherapy, crude medicines, and alternative therapies have been utilized in homes and hospitals, additionally to modern medicine; these also play a vital role in preventive medicine (Inoue, Hayashi, \& Craker, 2019). People with Alzheimer's disease experience a loss of appetite. Therapeutic plants, therefore, maintain the stimulation of appetites such as lemon, lime, nutmeg, clove, coriander, orange, grapefruit, ginger, and cardamom oils (Bridges by epoch memory care assisted living, n.d.). Apart from memory loss, patients with dementia also suffer from agitation and aggression, which are listed as the most complicated symptoms to cure. Essential oils, in particular lavender, bergamot, and lemon, have been validated by researchers in alleviating these symptoms (Laguipo, 2021). Hence, essential oils promote tranquility, additionally help to soothe anxiety and mood (Vineyard memory care community, 2019).
Some studies have established that lavender utilizing aromatherapy can enhance performance and relieve insomnia in patients with dementia (Upham, 2020). With its calming scent, lavender is widely recognized for subsiding depression, turbulent emotions, and irritation (Natural Health Practitioners of Canada, 2020). One caregiver professes that utilizing lavender within terms of comforting sleep deprivation for her patient provided positive results. Lavender sachets beneath the pillow benefited individuals with dementia, in a clinical trait in the United Kingdom (UK), to sleep better (Bridges by epoch memory care assisted living, n.d.).

Properties of lavender essential oil are scientifically reported to accommodate local anesthetic, sedative, analgesic, anticonvulsant, antispasmodic, cholinesterase inhibitory, antioxidant, antibacterial, and antifungal effects and inhibition of microbial resistance (AliShtayeh, Abu-Zaitoun, Dudai, \& Jamous, 2020).

Rosemary, referred to as Rosmarinus officinalis $L$., is proved to be a memory booster that precludes loss of cognitive and memory functions in Alzheimer's. Approximately a quarter of the elderly with the age of 80 and above are found to suffer from these symptoms (Habtemariam, 2016; AromaCare, 2015). Other rosemary essential oils components, including essential oils constituents, have been recognized to influence brain function at therapeutic doses. When investigated in the human body utilizing the scopolamineinduced dementia model of Alzheimer's disease, the crude extract of rosemary was shown to ameliorate memory loss (Habtemariam, 2016). As memory loss is one of the most serious concerns of dementia, rosemary is a vital component of cognitive impairment therapy (Laguipo, 2021). Rosemary is known for a boosting oil that triggers the human brain and body as well as peppermint; in addition, cognitive behavior and emotions could be evolved. Constipation, depression's signs, and 
appetite regulation can be relieved by applying rosemary through inhalation, diffusion in a room, or atomizer (Habtemariam, 2016).

Bergamot essential oil may help alleviate agitation, one of the most prevalent symptoms, in patients with Alzheimer's disease and dementia. Hence, unlike other dementia medicines, this alleviation occurs without the sedative effect. With its ability to reduce diastolic and systolic blood pressure, bergamot plays a vital role in soothing anxiety and stress during aromatherapy. Bergamot candlelight is the simplest approach to aromatherapy. A bergamot candle can rescue your relaxation and mood. Instead of utilizing candlelight when candles are not obtainable, reed diffusers are an alternative choice. A vase is filled with essential oils, and the thin reeds are placed with their ends in the essential oils. The reeds absorb the essential oil and consequently releasing the scent progressively in the air. This furnishes longlasting aromatherapy, which suits in a small place. In addition to incense and room sprays, aromatherapy can be conducted with a nebulizer and ultrasonic diffusers (KOLBECK, 2021). Demented patients frequently are unable to physically express their pain which subsequently draws to untreated symptoms and conduces to agitation. Preclinical confirmation of analgesic properties has been extensively contributed by bergamot essential oil. Moreover, the essential oil of bergamot causes anxiolytic-like effects devoid of sedation, commonly recognized as benzodiazepines, and has a significant beneficial impact for demented patients (Scuteri-Corasaniti, 2019). Unlike other oils, bergamot is known to slow down heart rate, decrease blood pressure, and modulate neural pathways in the brain, easing insomnia (Sleep Better Georgia dental sleep medicine, 2019).

There is an abundance of studies that have been established regarding the lemon oils for aromatic ambition. Its specialties to relieve anxiety, sleep deprivation, and memory impairment make lemon balm one of the most effective herbs (Natural Health Practitioners of Canada, 2020). Lemon Balm, commonly known as Melissa officinalis, is one of the essential oils that have been researched associated with Alzheimer's disease and dementia. It benefits tranquillity and relaxation, which assists individuals with anxiety and insomnia. Lemon Balm has been analyzed the most for its impacts on Alzheimer's patients (Schmid, 2010). Compared to other essential oils, lemon oils function effectively and are costly. It may develop their memories and relieve indigestion. To use lemon oils for the healing process, drip it into a bath, inhale directly, diffuse, spray, or apply straight to the skin (Sauer, 2015). Besides, Professor Elaine Perry, Prof. of Neurochemical Pathology, Inst. For Aging and Health, Newcastle General Hospital, United Kingdom, claims that lemon balm, or Melissa, enhances mood and diminishes agitation in patients with Alzheimer's disease; additionally, it raises the quality of life.

The majority of essential oils are safe to utilize; however, there are some cautions and negative impacts to consider before using them, in particular, if you adhere to a medical prescription. In addition, Awareness should be determined on pregnant or breastfeeding women and young children while applying essential oils. Those people should be under the care of a doctor, ensure that they exploit the essential oils appropriately, and never take it through ingestion.

When taken with essential oils by inhaling aromatherapy, it is conceivable that there would be no harm to the human body (Lemke, RN, CCAP, \& Health and Wellness Options LLC, n.d.; Cronkleton, 2019; Halcón, PhD, MPH, RN, \& RATC, n.d.). Nevertheless, some precautions might have to be taken into consideration, particularly in children and pregnant women (Capritto, 2020). 


\section{CASE STUDY}

\subsection{Amyloid plaques with Alzheimer's disease}

Brain tissues associated with a dead woman with an aberrant mental illness have been found with a formation of clumps and tangled bundles of fibers (NIA, 2021). Furthermore, scientists have discovered uncommon genetic mutations in a few hundred extended families intercontinental, ensuring that people will acquire Alzheimer's disease. In any of three genes, these mutations can arise-biological processes engaged in each of these genes relate to the aggregation and production of beta-amyloid. According to the estimates, these mutations are found in approximately 1 percent of Alzheimer's patients. With modern technology, scientists conducted an experiment where mice are genetically engineered to have some of these mutated genes to test whether or not these genetic mutations would develop into Alzheimer's disease. The results are found that amyloid plaques are accumulated in the mice's brain as the genes are mutated. This results in memory impairment, affecting the mice not to be able to recognize a way through the maze. Moreover, these mice also develop symptoms associated with Alzheimer's disease in humans. Researchers have shown that three chromosomes that carry the APP gene are replicated in Down syndrome patients instead of two in people with normal conditions. The investigations propose that not all patients with Down syndrome will rise into Alzheimer's; nonetheless, approximately three fourth of those individuals with the age more than 65 are predicted to have Alzheimer's (Alzheimer's Association, 2017).

\subsection{Effectiveness of essential oils on individuals}

The researchers have analyzed the effects of aromatherapy impacting the elderly in which few of them experienced Alzheimer's disease; the conclusions were carried out for 28 days. During the investigation, rosemary and lemon essential oils were utilized in the morning, whereas lavender and orange were applied in the evening. Subsequently, the upshots of the study depict and explain the positive outcomes of aromatherapy on volunteer's cognition induces. Furthermore, the studies of 20 healthy individuals were performed to investigate how 1,8-cineole, which is contained in rosemary essential oil, impacts individuals' cognitive performance and mood. Both individuals' cognitive indices and mood alteration were evaluated in scores before and after the experiment. In addition, the participants' venous blood was collected at the end of the investigation to evaluate the presence of 1,8-cineole in the serum. The results demonstrated that 1,8 cineole in the rosemary essential oil induced the ability to accomplish tasks as the participants' speed and accuracy improved. Thus, even though the ability to modulate mood was not practically hown, the aim of the experiment was successfully achieved (Filiptsova, Gazzavi-Rogozina, Timoshyna, Naboka, Dyomina, \& Ochkur, 2017).

\section{CONCLUSION}

The most frequent cause of dementia in the elderly is Alzheimer's disease. Dementia is defined by a loss of cognitive capacities, such as the ability to memorize, reason, and analyze things logically. However, Alzheimer's disease is a syndrome, not a disease. Mixed dementia occurs when multiple types of dementia appear at the same time. The only way to confirm the diagnosis and treatment of mixed dementia is by autopsy. Alzheimer's disease affects the way that the brain functions which controls thought, memory, and language. Anxiety, irritability, a lack of inventiveness, mild obliviousness, time and location obstacles, and communication difficulty are some of the symptoms which patients may experience and they may develop gradually. Patients with Alzheimer's usually contain low levels of neurotransmitters chemical messengersonce amyloid and tau damage the brain cells. Various parts of the brain, particularly 
the first section where memories are stored, are weakened. As the brain is permanently damaged, Alzheimer's disease causes brain cells to degrade, lose their connections, and eventually die. The question of whether amyloid and tau cause disease or are just a part of the disease development is currently being discussed. Patients with Alzheimer's disease suffer a lack of appetite. Essential oils, notably lavender, bergamot, and lemon, have been shown in studies to alleviate these symptoms. These oils also assist in alleviating anxiety and improve mood in people suffering from the condition. Although most essential oils are safe to use, there are certain restrictions and adverse effects to be aware of, such as a pregnant or breastfeeding mother. Scientists have identified rare genetic mutations in a few hundred extended families around the world, contributing to accumulation and synthesis of beta-amyloid. To clarify, mice were genetically modified to carry some of these faulty genes. As a result, the mice's memory is impaired together with other symptoms resembling the disease. Aromatherapy's impact on the elderly with Alzheimer's disease was studied by researchers. The researchers looked at how the compound 1,8-cineole, which is found in rosemary essential oil, affects people's cognitive abilities and emotions. The results showed that 1-8-Cineole in rosemary oil increased the participants' capability of completing tasks by increasing their agility and accuracy.

\section{Acknowledgement: None}

\section{Conflict of Interest: None}

\section{Source of Funding: None}

\section{REFERENCES}

1. A Breakdown of All the Best Essential Oils for Sleep-Sleep Better Georgia Blog. (2019, September 12). Sleep Better Georgia Dental Sleep Medicine. https://www. sleepdunwoody.com/blog/2019/09/12/abreakdown-of-all-the-best-essential-oils-forsleep/
2. Ali, B., Al-Wabel, N. A., Shams, S., Ahamad, A., Khan, S. A., \& Anwar, F. (2015, August 1). Essential oils used in aromatherapy: A systemic review. Science Direct. https://www.sciencedirect.com/ science/article/pii/S2221169115001033\#tbl 1

3. Ali-Shtayeh, M. S. (2020, February 7). Downy Lavender Oil: A Promising Source of Antimicrobial, Antiobesity, and AntiAlzheimer's Disease Agents. Hindawi. https://www.hindawi.com/journals/ecam/20 20/5679408/

4. Alzheimer's and Hallucinations, Delusions, and Paranoia. (2017, May 17). National Institute on Aging. https://www.nia.nih.gov/ health/alzheimers-and-hallucinationsdelusions-and-paranoia

5. Alzheimer's Association. (2017, March). Beta-amyloid and the amyloid hypothesis. https://www.alz.org/national/documents/topi csheet_betaamyloid.pdf

6. Alzheimer's Association. (n.d.). What is Alzheimer's Disease?. Retrieved August 07, 2021, from https://www.alz.org/alzheimersdementia/what-is-alzheimers

7. AromaCare. (2015, September 21). How to fight against Alzheimer's with Rosemary essential oils? AromaCare. https://aromacare.fr/en/how-to-fight-against-alzheimerswith-rosemary-essential-oils/

8. Aromatherapy: Do Essential Oils Really Work? (n.d.). Johns Hopkins Medicine. Retrieved August 07, 2021, from https://www.hopkinsmedicine.org/health/we llness-and-prevention/aromatherapy-doessential-oils-really-work

9. Aromatherapy for Alzheimer's patients. (n.d.). Bridges ${ }^{\circledR}$ by EPOCH. Retrieved August 18, 2021, from https://www. bridgesbyepoch.com/resources/educationalresources/aromatherapy-alzheimer\%E2\% 80\%99s-patients-1497443677

10. BrightFocus Foundation. (2019, December 21). Amyloid Plaques and Neurofibrillary Tangles. https://www.brightfocus.org/ alzheimers-disease/infographic/amyloidplaques-and-neurofibrillary-tangles

11. Capritto, A. (2020, November 4). The dangers of essential oils: Why natural isn't always safe. CNET. https://www.cnet.com/ health/are-essential-oils-actually-safe/

12. Cronkleton, E. (2019, March 8). Aromatherapy Uses and Benefits. 
Healthline. https://www.healthline.com/ health/what-is-aromatherapy

13. Dementia Specialist Admiral Nurses. (2019, June). Aromatherapy. Retrieved August 07, 2021, from https://www.dementiauk. org/wp-content/uploads/2019/06/

Aromatherapy-new-template-WEB.pdf

14. Filiptsova, O. V., Gazzavi-Rogozina, L. V., Timoshyna, I. A., Naboka, O. I., Dyomina, Y. V., \& Ochkur, A. V. (2017, May 8). The essential oil of rosemary and its effect on the human image and numerical short-term memory. Science Direct. https://www. sciencedirect.com/science/article/pii/S23148 08X16301890

15. Habtemariam, S. (2016, January 28). The Therapeutic Potential of Rosemary (Rosmarinus officinalis) Diterpenes for Alzheimer's Disease. Hindawi. https://www.hindawi.com/journals/ecam/20 16/2680409/

16. Halcón, L., PhD, MPH, RN, \& RATC. (n.d.). Are Essential Oils Safe? Taking Charge of Your Health \& Wellbeing. Retrieved August 18, 2021, from https://www.takingcharge.csh.umn.edu/areessential-oils-safe

17. Jimbo, D., Kimura, Y., Taniguchi, M., Inoue, M., \& Urakami, K. (2010, March 8). Effect of aromatherapy on patients with Alzheimer's disease. Wiley Online Library. https://onlinelibrary.wiley.com/doi/full/10.1 111/j.1479-8301.2009.00299.x

18. Leonard, W. (2018, February 8). Alzheimer's Disease Prevention. Healthline. https://www.healthline.com/health/alzheime rs-disease-prevention\#diet

19. Kolbeck, S. (2021, May 21). Bergamot essential oil benefits. Chicagotribune.Com. https://www.chicagotribune.com/consumerreviews/sns-bestreviews-wellnessbergamot-essential-oil-benefits-20210521efveqj5v2zbzpfu7hdlk2eu2wi-story.html

20. Laguipo, A. B. B. B. (2021, February 18). Aromatherapy for Dementia. NewsMedical.Net. https://www.news-medical. net/health/Aromatherapy-for-Dementia.aspx

21. Leinonen, V., Koivisto, A., Savolainen, S., Rummukainen, J., Tamminen, J., Tillgren, T., Vainikka, S., Pyykkö, O., Mölsä, J., Fraunberg, M., Pirttilä, T., Jääskeläinen, J., Soininen, H., Rinne, J., \& Alafuzoff, I. (2010, October 25). Amyloid and tau proteins in cortical brain biopsy and Alzheimer's disease. Wiley Online Library. https://onlinelibrary.wiley.com/doi/abs/10.1 002/ana.22100

22. Lemke, B., RN, CCAP, \& Health \& Wellness Options LLC. (n.d.). Benefits of Using Aromatherapy. ALzheimer's Association. Retrieved August 18, 2021, from https://act.alz.org/site/DocServer/ M3_Benefits_of_Using_Aromatherapy_Sup port_for_Caregivers.pdf

23. LEMON BALM: Overview, Uses, Side Effects, Precautions, Interactions, Dosing and Reviews. (n.d.). Natural Medicines Comprehensive Database Consumer Version. Retrieved August 07, 2021, from https://www.webmd.com/vitamins/ai/ingred ientmono-437/lemon-balm

24. Maiko Inoue, Shinichiro Hayashi and Lyle E. Craker (February 4th 2019). Role of Medicinal and Aromatic Plants: Past, Present, and Future, Pharmacognosy Medicinal Plants, Shagufta Perveen and Areej Al-Taweel, IntechOpen, DOI: 10.5772/intechopen.82497. Available from: https://www.intechopen.com/chapters/6547 7

25. Natural Health Practitioners of Canada. (2020, January 28). Aromatherapy for Alzheimer's Disease. https://www.nhpcanada.org/blog/articles.ht $\mathrm{ml}$ ?id=91\&title=Aromatherapy-for Alzheimer\%E2\%80\%99s-Disease

26. NHS. (2021, July 5). Causes Alzheimer's Disease. Nhs.Uk. https://www.nhs.uk/ conditions/alzheimers-disease/causes/

27. NIH National Institute on Aging [NIA]. (2021, July 08). Alzheimer's Disease Fact Sheet. Retrieved August, 07, 2021, from https://www.nia.nih.gov/health/alzheimersdisease-fact-sheet

28. Ring, T. (2019, April 19). The Benefits of Aromatherapy and Essential Oils for Memory Care. Vineyard Henderson. https://www.vineyardhenderson.com/aromat herapy-essential-oils-for-memory-car/

29. Sauer, A. (2015, August 10). Manage Dementia's Side Effects with These 7 Essential Oils. Alzheimers.Net. https:// www.alzheimers.net/10-10-14-essentialoils-dementia

30. Schmid, J. (2010, August 5). Can Aromatherapy Help Alzheimer's Disease. Best Alzheimer's Products. https://bestalzheimers-products.com/alternativetherapy-for-alzheimers/aromatherapy-foralzheimers 
31. Scuteri, D., Rombola, L., Morrone, L. A., Bagetta, G., Sakurada, S., Sakurada, T., Tonin, P., \& Corasaniti, M. T. (2019, July 6). Neuropharmacology of the Neuropsychiatric Symptoms of Dementia and Role of Pain: Essential Oil of Bergamot as a Novel Therapeutic Approach. PubMed Central (PMC). https://www.ncbi.nlm.nih. gov/pmc/articles/PMC6651821/

32. Southern Cross Medical Library. (2020, June). Alzheimer's disease-causes, symptoms, prevention. Southern Cross. https://www.southerncross.co.nz/group/med ical-library/alzheimers-disease-causessymptoms-prevention

33. The Healthline Editorial Team. (2020, September 1). Dementia and Alzheimer's: What Are the Differences? Healthline. https://www.healthline.com/health/alzheime rs-disease/difference-dementia-alzheimers \#dementia

34. Upham, B., \& Sinha, S., MD. (2020, March 29). What Essential Oils Are Good for Alzheimer's Disease? | Everyday Health.
EverydayHealth.Com.

https://www.everydayhealth.com/alzheimers -disease/what-you-need-to-know-aboutessential-oils-and-alzheimers-disease/

35. What Happens to the Brain in Alzheimer's Disease? (2017, May 16). National Institute on Aging. https://www.nia.nih.gov/health/ what-happens-brain-alzheimers-disease

36. 6 Tips for Planting an Alzheimer's Sensory Garden | Five Star Senior Living. (2020, November 20). Five Star Senior Living. https://www.fivestarseniorliving.com/blog/6 -tips-for-planting-an-alzheimers-sensorygarden

How to cite this article: Hemangkorn N, Phummai P, Punyacharoen P. Effects of essential oils and aromatic plants on Alzheimer's disease and dementia. International Journal of Science \& Healthcare Research. 2021; 6(3): 350-358. DOI: https://doi.org/ 10.52403/ijshr.20210760 